\title{
Coexistencia de trastornos gastrointestinales funcionales en lactantes y preescolares latinoamericanos
}

\section{Coexistence of functional gastrointestinal disorders in Latin American infants and preschoolers}

\author{
Carlos A. Velasco-Benítez ${ }^{*}$, Ricardo Chanis², Edgar Játiva³ ${ }^{3}$ Milton Mejia4a, Jairo Moreno ${ }^{5}$, Carmen R. Ramírez-Hernández ${ }^{6}$,
} Eder A. Villamarín-Betancourt?, José F Gómez-Urrego ${ }^{8}$

\footnotetext{
1 Profesor titular Universidad del Valle, Cali, Colombia.

2 Gastroenterólogo Pediatra, Servicio de

Gastroenterología Pediátrica y Endoscopia Digestiva Hospital del Niño Dr. José Renán Esquivel, Ciudad de Panamá, Panamá.

3 Pediatra Universidad Central del Ecuador, Quito,

Ecuador. Universidad de La Frontera, Chile.

${ }^{4}$ Gastroenterólogo Pediatra, Hospital Infantil de Nicaragua Manuel de Jesús Rivera, Managua, Nicaragua.

5 Pediatra Clínica Pediátrica Colsanitas, Bogotá, Colombia.

${ }^{6}$ Pediatra Hospital Regional María Inmaculada, Florencia, Colombia.

7 Pediatra Universidad del Valle, Universidad Santiago de Cali, Cali, Colombia.

8 Pediatra, coordinador de posgrado en Pediatría, Universidad Libre, profesor Pontificia Universidad Javeriana, Cali, Colombia.

*Correspondencia: Carlos A. Velasco-Benítez, carlos.velasco@correounivalle.edu.co
}

Fecha recibido: $\quad 08 / 02 / 18$ Fecha aceptado: 29/04/19

\begin{abstract}
Resumen
Introducción: se ha descrito la coexistencia de trastornos gastrointestinales funcionales (c-TGF) en lactantes y preescolares (LactPre); sin embargo, hay poca literatura sobre los principales c-TGF y sus características. Objetivo: describir la prevalencia y posibles asociaciones de c-TGF en un mismo LactPre latinoamericano. Metodología: estudio de prevalencia en LactPre de Colombia, Ecuador, Nicaragua y Panamá de consulta externa y urgencias, a quienes, según los criterios de Roma III en español, se identificaron regurgitación, síndrome de rumiación, síndrome del vómito cíclico, cólico, diarrea funcional, estreñimiento funcional y disquecia. Se tuvieron en cuenta la edad, el sexo y el origen. La estadística incluyó t de Student, chi cuadrado, prueba exacta de Fisher, análisis uni y multivariados y el cálculo de los OR e IC 95\%, siendo una $p<0,05$

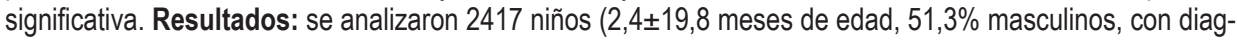
nóstico de por lo menos algún TGF del 35,7\%). Se presentó c-TGF en el 3,6\%, siendo el principal el síndrome de rumiación del lactante (SRL) más estreñimiento funcional (EF). Hubo predomino del género masculino en los lactantes y ser colombiano. Conclusión: la principal c-TGF en este grupo de LactPre de Latinoamérica es el SRL más EF, presentándose principalmente en el sexo masculino y en lactantes menores de 24 meses.
\end{abstract}

\section{Palabras clave}

Lactantes, preescolares, prevalencia, enfermedades gastrointestinales.

\begin{abstract}
Introduction: Coexistence of functional gastrointestinal disorders (FGID) in infants and preschool children has been described, but there is little literature on the main types of FGID coexistence or their characteristics. Objective: This study describes the prevalence and possible associations of FGID coexistence among Latin American infants and preschool children. Methodology: This is a prevalence study of infants and preschool children conducted in Colombia, Ecuador, Nicaragua and Panama. Children included were outpatients and emergency patients who were identified according to the Rome III Criteria in Spanish as suffering from regurgitation, rumination syndrome, cyclic vomiting syndrome, colic, functional diarrhea, functional constipation and/ or dyspepsia. Age, sex and origin of patients were registered. Statistical analyses included Student's T test, chi squared test, Fisher's exact test, univariate analysis, multivariate analysis and calculation of odds ratios and $95 \%$ confidence intervals with $p<0.05$ set as significant. Results: Two thousand four hundred and seventeen children were included. Their age range was 2.4 months to 19.8 months of age, and $51.3 \%$ were male. The proportion of patients with a diagnosis of at least one FGID was $35.7 \%$. FGID coexistence was found in $3.6 \%$ of the patients. The most frequent combination was rumination syndrome plus functional constipation. There were predominances of males, infants and Colombian children in the total sample. Conclusion: The most commonly coexisting FGIDs in this group of Latin America infants and preschool children were infant rumination syndrome and functional constipation which were found together most frequently among boys who were under 24 months old.
\end{abstract}

\section{Keywords}

Infants, pre-school, prevalence, gastrointestinal diseases. 


\section{INTRODUCCIÓN}

Según los criterios de Roma IV, los trastornos gastrointestinales funcionales (TGF) en lactantes y preescolares se definen como una combinación diversa y variable de síntomas gastrointestinales recurrentes o crónicos que, después de una adecuada evaluación médica, no son atribuibles a otras condiciones médicas (1). A nivel mundial, la prevalencia de TGF, según los criterios de Roma III, en niños entre los 0 y 4 años oscila entre 27,1\% y 38,0\% (2). Recientemente, en Estados Unidos, Robin y colaboradores describieron una prevalencia del $24,7 \%$, según los criterios de Roma IV, en menores de 3 años (3). En lactantes y preescolares latinoamericanos (latam) se ha descrito una prevalencia de estos TGF entre 11,6\% y 47,8\%, según los criterios de Roma III en español, dependiendo del sitio donde se haya realizado el estudio: $47,8 \%$ en pacientes hospitalizados, $38,2 \%$ en un servicio de urgencias pediátricas, $26,6 \%$ en consulta externa particular y hospitalaria y $11,6 \%$ en consulta externa de crecimiento y desarrollo del niño sano (4).

A pesar de que se describe la coexistencia de estos TGF, hay poca literatura sobre los principales TGF coexistentes (c-TGF) y las características de este grupo de niños. En escolares y adolescentes latam se presenta c-TGF en un $8,4 \%$, siendo la principal coexistencia el síndrome de intestino irritable más dolor abdominal funcional, con predominio del género femenino (5). La importancia de estudiar esta coexistencia en los TGF radica en que aportará un mejor entendimiento de la fisiopatología y patogénesis del modelo biopsicosocial de los TGF en este grupo etario, desde lo genético, nutricional, ambiental, psicosocial, cultural, socioeconómico e infeccioso, entre otros, para así definir mejor la epidemiología, los síntomas, la comorbilidad y la calidad de vida relacionada con la salud de los lactantes y preescolares con TGF.

El objetivo de este trabajo es describir la prevalencia y posibles factores de riesgo en lactantes y preescolares latinoamericanos de presentar c-TGF.

\section{MATERIAL Y MÉTODO}

Se trata de un estudio descriptivo observacional no experimental de corte transversal de tipo prevalencia realizado entre el 1 de mayo y el 31 de octubre de 2015 con la base de datos de Functional International Digestive Epidemiological Research Survey Group (FINDERS), un grupo de investigación transnacional conformado por varios integrantes de la Sociedad Latinoamericana de Gastroenterología, Hepatología y Nutrición Pediátrica (SLAGHNP).

Los métodos de recolección de datos fueron iguales en todos los países participantes: Colombia, Ecuador, Nicaragua y Panamá. Se entrevistaron a los padres o los acudientes latam que firmaron un consentimiento informado de niños menores de 4 años de una consulta externa ambulatoria y hospitalaria de control y desarrollo del niño sano de Colombia, Nicaragua y Panamá, y de una consulta de urgencias del Ecuador. Las variables sociodemográficas que se tuvieron en cuenta fueron la edad, el sexo y el país de origen. Fueron excluidos por historia conocida los niños con trastornos gastrointestinales orgánicos, como enfermedad por reflujo gastroesofágico, alergia a la proteína de la leche de vaca, enfermedad de Hirschsprung, parálisis cerebral, reflujo vesicoureteral, síndrome convulsivo y enfermedad cardíaca. Para identificar los TGF se utilizó el cuestionario para síntomas gastrointestinales pediátricos Roma III para lactantes y preescolares (QPGS-III), el cual se ha validado y probado en español (6). Según los lineamientos del Scoring Instructions for Infant/Toddler Report Form for the Rome III Diagnostic Questionnaire on Pediatric Gastrointestinal Symptoms for Infants and Toddlers, los TGF que se identificaron fueron el cólico del lactante (0-4 meses), la disquecia del lactante (0-5 meses), la regurgitación del lactante (0-12 meses), el síndrome de rumiación del lactante (0-24 meses), el síndrome de vómito cíclico (0-48 meses), la diarrea funcional (0-48 meses) y el estreñimiento funcional (0-48 meses) (7). Para fines de este estudio, y que a futuro permita comparar estos resultados con la nueva clasificación de los criterios de Roma IV, se tuvieron en cuenta todas las posibles superposiciones (1).

Los grupos de edad tenidos en cuenta fueron los lactantes (entre los 0 y 24 meses de edad) y los preescolares (entre los 2 y 4 años). Se consideró c-TGF cuando se presentaron 2 o más TGF en un mismo niño. El estudio fue aprobado por el Comité de Ética de la Universidad del Valle de Cali, Colombia.

Ante la posibilidad de errores de transcripción se revisaron el $10 \%$ de los datos y luego se compararon con las formas originales. El análisis estadístico por medio de Stata (Stata 15 software; StataCorp, College Station, TX) incluyó la t de Student a dos colas, el chi cuadrado y la prueba exacta de Fisher. Para los posibles factores de riesgo para c-TGF se realizaron análisis uni y multivariados y el cálculo de los OR se llevó a cabo entre la variable de exposición de interés (sexo, edad, origen) y la variable efecto (presencia o ausencia de c-TGF). Una $p<0,05$ se consideró como estadísticamente significativa.

\section{RESULTADOS}

Se analizaron 2417 niños de Colombia $(\mathrm{n}=1694)$, Ecuador $(\mathrm{n}=322)$, Nicaragua $(\mathrm{n}=203)$ y Panamá $(\mathrm{n}=198)$, de $2,4 \pm 19,8$ meses de edad (rango de 1 a 48 meses). 67,1\% fueron lactantes entre 1 y 24 meses y $51,3 \%$ del sexo masculino, con diagnóstico de por lo menos algún TGF del 35,7\%, siendo el principal TGF en todas las edades el estreñimiento 
funcional (19,7\%); en lactantes, el síndrome de rumiación del lactante $(7,2 \%)$; y en preescolares, el síndrome de vómito cíclico (4,0\%), datos que se observan en la Tabla 1.

En la Tabla 2 se observa la coexistencia de TGF en un mismo niño de los 2417 niños latam estudiados en todas las edades en el 3,6\%; en lactantes en el 3,5\% y en preescolares en el $0,2 \%$ ( $3,3 \%$ con 2 TGF y $0,4 \%$ con 3 TGF), siendo las principales coexistencias el síndrome de rumiación del lactante más estreñimiento funcional $(1,0 \%)$ y la regurgitación más disquecia más estreñimiento funcional $(0,1 \%)$.

Las posibles asociaciones se observan en la Tabla 3. Hubo predomino del género masculino (OR 1,84; IC 95\% $1,134-3,02 ; p=0,0083$ ), de los lactantes (OR 10,52; IC $95 \% 3,88-39,87 ; p 0,0000)$ y ser originario de Colombia (OR 6,85; IC 95\% 1,78-58,37; $p$ 0,0021) cuando se presentó coexistencia de más de un TGF en el mismo niño.

\section{DISCUSIÓN}

\section{Prevalencia de TGF en lactantes y preescolares}

En estos 4 países latinoamericanos, la prevalencia de presentar algún TGF por los criterios de Roma III en español fue del $35,7 \%$, inferior a lo descrito por Rouster y colaboradores; del 52,0\% en Estados Unidos y según lo reportado por Chogle y colaboradores; del 40,0\% y 40,5\% en países latinoamericanos; y prevalencia superior a los resultados de van Tilburg y colaboradores del 27,1\% en Estados Unidos (8-11). Vandenplas y colaboradores encontraron que para médicos expertos a nivel mundial, las prevalencias más probables son la regurgitación en el 30,0\%, el cólico en el 20,0\% y el estreñimiento funcional en el 15,0\% (12). En este estudio, el principal TGF en lactantes fue el síndrome de rumiación del lactante, diferente a lo descrito por van Tilburg y colaboradores y Rouster y colaboradores en Estados Unidos, donde lo más frecuente fue la regurgitación; y a lo reportado por Chogle y colaboradores en países latam, donde lo más común fue el cólico del lactante $(9,10)$.

En preescolares, en estos 4 países latam estudiados, el principal TGF fue el estreñimiento, al igual que en Estados Unidos y en otros países latam (8-11). Recientemente, según los criterios de Roma IV, Robin y colaboradores describieron una prevalencia para presentar algún TGF del 24,7\% en Estados Unidos, siendo lo más frecuente en lactantes la regurgitación y para preescolares el estreñimiento (3). La variabilidad en estas prevalencias es dependiente de la región desde donde se realizan los estudios, en donde se involucran factores genéticos, nutricionales, ambientales, psicosociales, culturales, socioeconómicos e infecciosos, entre otros, propios de cada país.

Tabla 1. Características generales de lactantes y preescolares latinoamericanos $(\mathrm{N}=2417)$

\begin{tabular}{|c|c|c|c|c|c|}
\hline & Latam & Colombia & Ecuador & Nicaragua & Panamá \\
\hline Total & 2417 & 1694 & 322 & 203 & 198 \\
\hline Edad (meses) (X DS) & $19,8(15,0)$ & $19,5(15,3)$ & $20,9(13,7)$ & $13,9(8,9)$ & $26,6(16,2)$ \\
\hline Lactante (1-12 meses) (n \%) & $1621(67,1)$ & $1153(68,1)$ & $203(63,0)$ & $186(91,6)$ & $79(39,9)$ \\
\hline Preescolar (13-48 meses) ( $\mathrm{n} \%)$ & $796(32,9)$ & $541(31,9)$ & $119(37,0)$ & $17(8,4)$ & $119(60,1)$ \\
\hline \multicolumn{6}{|l|}{$\operatorname{Sexo}(n \%)$} \\
\hline Femenino & $1179(48,8)$ & $825(48,7)$ & $149(46,3)$ & $107(52,7)$ & $98(49,5)$ \\
\hline Masculino & $1238(51,2)$ & $869(51,3)$ & $173(53,7)$ & $96(47,3)$ & $100(50,5)$ \\
\hline \multicolumn{6}{|l|}{ TGF $(n \%)$} \\
\hline Ausente & $1555(64,3)$ & $1008(59,5)$ & $218(67,7)$ & $176(86,7)$ & $153(77,3)$ \\
\hline Presente & $862(35,7)$ & $686(40,5)$ & $104(32,3)$ & $27(13,3)$ & $45(22,7)$ \\
\hline Regurgitación del lactante $(\mathrm{n} \%)^{\star * *}$ & $89(3,7)$ & $57(3,4)$ & $10(3,1)$ & $16(7,9)$ & $6(3,0)$ \\
\hline Síndrome de rumiación del lactante $(\mathrm{n} \%)^{\star *}$ & $117(4,8)$ & $110(6,5)$ & $1(0,3)$ & $4(2,0)$ & $2(1,0)$ \\
\hline Síndrome de vómito cíclico $(\mathrm{n} \%)^{*}$ & $81(3,4)$ & $78(4,6)$ & $2(0,6)$ & $1(0,5)$ & $0(0,0)$ \\
\hline Cólico del lactante $(\mathrm{n} \%)^{* * * * *}$ & $41(1,7)$ & $38(2,2)$ & $0(0,0)$ & $0(0,0)$ & $3(1,5)$ \\
\hline Diarrea funcional $(\mathrm{n} \%)^{*}$ & $24(1,0)$ & $22(1,3)$ & $0(0,0)$ & $2(1,0)$ & $0(0,0)$ \\
\hline Estreñimiento funcional $(\mathrm{n} \%)^{*}$ & $475(19,7)$ & $354(20,9)$ & $87(27,0)$ & $3(1,5)$ & $31(15,7)$ \\
\hline Disquecia del lactante $(\mathrm{n} \%)^{\star \star * *}$ & $35(1,5)$ & $27(1,6)$ & $4(1,2)$ & $1(0,5)$ & $3(1,5)$ \\
\hline
\end{tabular}

DS: desviación estándar; Latam: Latinoamérica; TGF: trastornos gastrointestinales funcionales; X: promedio.

${ }^{*} 0-48$ meses; ${ }^{* *} 0-24$ meses; ${ }^{* * *} 0-12$ meses; ${ }^{* * *} 0-5$ meses; ${ }^{* * * *} 0-4$ meses. 
Tabla 2. Coexistencia de trastornos gastrointestinales funcionales en lactantes y preescolares latinoamericanos $(\mathrm{N}=2417)$

\begin{tabular}{|c|c|c|c|c|c|}
\hline & Latam & Colombia & Ecuador & Nicaragua & Panamá \\
\hline Total & 2417 & 1694 & 322 & 203 & 198 \\
\hline TGF- & $1555(64,3)$ & $1008(59,5)$ & $218(67,7)$ & $176(86,7)$ & $153(77,3)$ \\
\hline TGF+ & $862(35,7)$ & $686(40,5)$ & $104(32,3)$ & $27(13,3)$ & $45(22,7)$ \\
\hline Sin overlap & $774(32,0)$ & $604(35,7)$ & $102(31,7)$ & $25(12,3)$ & $43(21,7)$ \\
\hline Con overlap & $88(3,7)$ & $82(4,8)$ & $2(0,6)$ & $2(1,0)$ & $2(1,0)$ \\
\hline Con 2 TGF & $79(3,3)$ & $75(4,3)$ & $1(0,3)$ & $2(1,0)$ & $1(0,5)$ \\
\hline srl_ef & $25(1,0)$ & $24(1,4)$ & $0(0,0)$ & $0(0,0)$ & $1(0,5)$ \\
\hline reg_col & $11(0,5)$ & $11(0,7)$ & $0(0,0)$ & $0(0,0)$ & $0(0,0)$ \\
\hline SVc_ef & $10(0,4)$ & $10(0,6)$ & $0(0,0)$ & $0(0,0)$ & $0(0,0)$ \\
\hline srl_col & $5(0,2)$ & $5(0,3)$ & $0(0,0)$ & $0(0,0)$ & $0(0,0)$ \\
\hline reg_srl & $3(0,1)$ & $2(0,1)$ & $0(0,0)$ & $1(0,5)$ & $0(0,0)$ \\
\hline srl_df & $2(0,08)$ & $1(0,06)$ & $0(0,0)$ & $1(0,5)$ & $0(0,0)$ \\
\hline reg_disq & $1(0,04)$ & $0(0,0)$ & $1(0,3)$ & $0(0,0)$ & $0(0,0)$ \\
\hline Con 3 TGF & $9(0,4)$ & $7(0,5)$ & $1(0,3)$ & $0(0,0)$ & $1(0,5)$ \\
\hline reg_disq_ef & $2(0,08)$ & $0(0,0)$ & $1(0,3)$ & $0(0,0)$ & $1(0,5)$ \\
\hline
\end{tabular}

COL: cólico del lactante; DF: diarrea funcional; DISQ: disquecia del lactante; EF: estreñimiento funcional; Latam: Latinoamérica; REG: regurgitación del lactante; SRL: síndrome de rumiación del lactante; SVC: síndrome del vómito cíclico; TGF: trastornos gastrointestinales funcionales.

Tabla 3. Posibles factores de riesgo en lactantes y preescolares latinoamericanos con coexistencia de trastornos gastrointestinales funcionales $(\mathrm{N}=862)$

\begin{tabular}{|c|c|c|c|c|c|}
\hline & \multicolumn{2}{|c|}{$20+$ TGF } & \multirow[b]{3}{*}{ OR } & \multirow[b]{3}{*}{ IC $95 \%$} & \multirow[b]{3}{*}{$P$} \\
\hline & No & Sí & & & \\
\hline & $774(89,8)$ & $88(10,2)$ & & & \\
\hline \multicolumn{6}{|l|}{ Grupos de edad } \\
\hline $\begin{array}{l}\text { Preescolar } \\
\text { (25-48 } \\
\text { meses) }\end{array}$ & 259 & 4 & 1,00 & & \\
\hline $\begin{array}{l}\text { Lactante } \\
\text { (0-24 meses) }\end{array}$ & 515 & 84 & 10,52 & $3,88-39,87$ & 0,0000 \\
\hline \multicolumn{6}{|l|}{ Sexo } \\
\hline Femenino & 388 & 31 & 1,00 & & \\
\hline Masculino & 386 & 57 & 1,84 & $1,14-3,02$ & 0,0083 \\
\hline \multicolumn{6}{|l|}{ País } \\
\hline Ecuador & 102 & 2 & 1,00 & & \\
\hline Colombia & 604 & 82 & 6,85 & $1,78-58,37$ & 0,0021 \\
\hline Nicaragua & 25 & 2 & 4,04 & $0,27-57,43$ & 0,1432 \\
\hline Panamá & 43 & 2 & 2,34 & $0,16-33,16$ & 0,3878 \\
\hline
\end{tabular}

TGF: trastornos gastrointestinales funcionales.

\section{Coexistencia}

Los criterios de Roma IV en lactantes y preescolares no discuten el tema de la coexistencia de TGF en el mismo paciente, a diferencia de los escolares y adolescentes, en quienes los estudios han demostrado que puede haber coexistencia de más de un trastorno de dolor abdominal funcional en un paciente individual, sin embargo, estos no relatan las características de los mismos $(5,13,14)$. En el presente estudio, la prevalencia para presentar 2 y 3 o más TGF en un mismo niño fue del $9,2 \%$ y $1,0 \%$, respectivamente, como se observa en la Tabla 4. van Tilburg y colaboradores, Rouster y colaboradores y Chogle y colaboradores reportaron prevalencias entre el 8,6\%-12,6\%; 5,0\%-13,0\% y $0,4 \%-4,6 \%$, respectivamente, de presentar 2,3 o más TGF en un mismo niño $(8,10,11)$.

Tabla 4. Comparación de prevalencias en lactantes y preescolares con coexistencia de trastornos gastrointestinales funcionales $(\mathrm{N}=2417)$

\begin{tabular}{lcccc}
\hline & $\begin{array}{c}\text { Velasco } \\
\mathbf{N}=\mathbf{2 4 1 7}\end{array}$ & $\begin{array}{c}\text { van } \text { Tilburg }^{10} \\
\mathbf{N = 2 6 4}\end{array}$ & $\begin{array}{c}\text { Chogle }^{9} \\
\mathbf{N}=1183\end{array}$ & $\begin{array}{c}\text { Rouster }^{7} \\
\mathbf{N}=\mathbf{3 3 2}\end{array}$ \\
\hline 2 TGF & $9,2 \%$ & $12,6 \%$ & $4,6 \%$ & $13,0 \%$ \\
3 o más TGF & $1,0 \%$ & $8,6 \%$ & $0,4 \%$ & $5,0 \%$ \\
\hline
\end{tabular}

TGF: trastornos gastrointestinales funcionales. 
Pocos estudios describen cuáles son los TGF que coexisten. Para el presente estudio, en el caso de coexistencia de 2 TGF, los principales fueron el síndrome de rumiación del lactante junto con el estreñimiento funcional. En lo referente a la coexistencia de 3 TGF, lo más frecuente fue la regurgitación, junto con la disquecia del lactante y el estreñimiento funcional. El estudio de Vandenplas y colaboradores no discrimina la coexistencia de los diferentes TGF, sin embargo, sí analiza la coexistencia de síntomas a nivel de la práctica clínica, como flatulencias, distensión abdominal, estreñimiento, diarrea, regurgitación y cólico, siendo las mayores combinaciones el cólico con la distensión abdominal, con la regurgitación y con la distensión abdominal (12).

En el presente estudio no se reporta coexistencia entre disquecia del lactante y estreñimiento funcional; sin embargo, Kramer y colaboradores reportaron una prevalencia para disquecia del lactante, según los criterios de Roma III, del $3,9 \%, 0,9 \%$ y $0,9 \%$, respectivamente, a los 1,3 y 9 meses de edad con 4 niños con coexistencia de disquecia del lactante y estreñimiento funcional al mismo tiempo (15). Con base en los criterios de Roma IV, Robin y colaboradores describieron un $9,6 \%$ de coexistencia de TGF (3).

\section{Asociaciones}

van Tilburg y colaboradores no encontraron diferencias estadísticas en género o raza, al igual que Robin y colaboradores; a diferencia del presente estudio, donde hubo predominio del género masculino y los lactantes entre $0 \mathrm{y}$ 2 años $(3,11)$.

Las fortalezas del estudio incluyen el gran tamaño de la muestra, además que se llevó a cabo en la consulta externa ambulatoria privada, hospitalaria y de urgencias de ciudades de varios países latinoamericanos de habla hispana. Asimismo, se empleó la misma metodología propuesta por FINDERS en todos los países, lo que permite su comparación.

Entre las limitaciones del estudio, a pesar de que incluye ciudades de varios países, no se puede descartar la posibilidad de que los resultados no pueden ser generalizados a toda Latinoamérica. Además, nosotros no realizamos una evaluación sistemática ni anamnesis en los niños encuestados y se podrían presentar diagnósticos médicos simultáneos que no se describen en el estudio. De la misma manera, no se preguntaron otros posibles factores de riesgo diferentes a los sociodemográficos, que puedan explicar el modelo biopsicosocial de esta entidad.

A futuro, y según los lineamientos de los criterios de Roma IV, se necesitan estudios epidemiológicos interculturales para determinar el impacto en la calidad de vida, así como investigaciones sobre la fisiopatología de la mayoría de los TGF, ya que este es poco conocido y se necesitan caracterizaciones de tipo genético, metabólico y neurofisiológico a nivel multicéntrico con grandes tamaños de muestra (1).

En conclusión, existe una baja prevalencia de c-TGF en un mismo lactante y preescolar en Latinoamérica, con predominio en el sexo masculino y en lactantes menores de 24 meses; siendo el síndrome de rumiación del lactante, junto con el estreñimiento funcional, las presentaciones más frecuentes, lo que invita a realizar estudios futuros que permitan ahondar más acerca de la coexistencia de los TGF en este grupo etario para su mejor entendimiento.

\section{REFERENCIAS}

1. Benninga M, Nurko S, Faure C, Hyman P, St James Roberts I, Schechter NL. Childhood functional gastrointestinal disorders: neonate/toddler. Gastroenterology. 2016;150(6):144355e2. doi: 10.1053/j.gastro.2016.02.016.

2. Ferreira-Maia A, Matijasevich A, Wang YP. Epidemiology of functional gastrointestinal disorders in infants and toddlers: a systematic review. World J Gastroenterol. 2016;22(28):6547-58. doi: https://doi.org/10.3748/wjg. v22.i28.6547.

3. Robin S, Keller C, Zwiener R, Hyman P, Nurko S, Saps $\mathrm{M}$, et al. Prevalence of pediatric functional gastrointestinal disorders utilizing the Rome IV Criteria. J Pediatr. 2018;195(4):134-9. doi: 10.1016/j.jpeds.2017.12.012.

4. Velasco-Benítez C, Saps M, Chanís R, Játiva E, Mejía M, Moreno J, et al. Desórdenes gastrointestinales funcionales en lactantes y preescolares latinoamericanos. Grupo gastroenterología, hepatología y nutrición pediátrica. Acta Gastroenterol Latinoam. 2018;48(1):126-30.

5. Velasco-Benítez C, Ramírez-Hernández C, Moreno-Gómez J, Játiva-Mariño E, Zablah R, Rodríguez-Reynosa L, et al. Superposición de desórdenes gastrointestinales funcionales en escolares y adolescentes latinoamericanos. Rev Chil Pediatr. 2018;89(6):726-31. doi: https://doi.org/10.4067/ S0370-41062018005000808.

6. Velasco-Benítez C, Sánchez-Pérez M, Aragón-Calvo L. Reability of the FINDERS questionnaire in Spanish based on Rome III criteria for functional gastrointestinal disorders in infants and toddler from Colombia, South America. J Pediatr Gastroenterol Nutr. 2015;61(9):S57.

7. Hyman P, Milla P, Benninga M, Davidson G, Fleisher D, Taminiau J. Childhood functional gastrointestinal disorders: neonate/toddler. Gastroenterology. 2006;130:151926. doi: 10.1053/j.gastro.2005.11.065.

8. Rouster A, Karpinski A, Silver D, Monagas J, Hyman P. Functional gastrointestinal disorders dominate pediatric gastroenterology outpatient practice. J Pediatr Gastroenterol Nutr. 2016;62(6):847-51. doi: 10.1097/ MPG.0000000000001023.

9. Chogle A, Velasco-Benitez C, Chanis R, Mejia M, Saps M. Multicountry cross-sectional study found that functional gastrointestinal disorders such as colic and functional 
dyschezia were common in South American infants. Acta Paediatr Int J Paediatr. 2018;107(4). doi: https://doi. org/10.1111/apa.14196.

10. Chogle A, Velasco-Benitez C, Koppen I, Moreno J, Ramírez Hernández C, Saps M. A population-based study on the epidemiology of functional gastrointestinal disorders in young children. J Pediatr. 2016;179(12):139-43.e1. doi: https:// doi.org/10.1016/j.jpeds.2016.08.095.

11. van Tilburg M, Hyman P, Walker L, Rouster A, Palsson O, Kim S, et al. Prevalence of functional gastrointestinal disorders in infants and toddlers. J Pediatr. 2015;166(3):684-9. doi: https://doi.org/10.1016/j.jpeds.2014.11.039.

12. Vandenplas Y, Abkari A, Bellaiche $M$, Benninga $M$, Chouraqui J, Çokuorap F, et al. Prevalence and health outcomes of functional gastrointestinal symptoms in infants from birth to 12 months of age. J Pediatr Gastroenterol
Nutr. 2015;61(5):531-7. doi: https://doi.org/10.1097/ MPG.0000000000000949.

13. Hyams J, Lorenzo D, Saps M, Shulman R, Staiano A, Tilburg $\mathrm{M}$, et al. Childhood functional gastrointestinal disorders: child/adolescent. Gastroenterology. 2016;150(6):1456-68. e2. doi: https://doi.org/10.1053/j.gastro.2016.02.015.

14. van Tilgurg M, Walker L, Palsson O, Kim S, Spiegel B, Spiller R. Prevalence of child/adolescent functional gastrointestinal disorders in a National U.S. Community Sample. Gastroenterology. 2014;146(5):143-4. doi: https://doi. org/10.1016/S0016-5085(14)60508-4.

15. Kramer E, Den Hertog-Kuijl J, van Den Broek L, van Leengoed E, Bulk A, Kneepkens C, et al. Defecation patterns in infants: a prospective cohort study. Arch Dis Child. 2015;100(6):533-6. https://doi.org/10.1136/archdischild-2014-307448. 